Although the idea of transurethral radical prostatectomy has been studied by others previously (Kavoussi et al.); this report of 2 patients with localized prostate cancer with negative margins using this novel NOTES approach may have been possible due to the great experience of the authors with laser prostate enucleation and new instrumentation for suturing endoscopically.

I am certain longer follow-up will determine the validity of this novel technique but undoubtedly, this innovating work has to be recognized as breaking ground.

Dr. Fernando J. Kim

Chief of Urology, Denver Health Med. Ctr.

Associate Professor, Univ. Colorado Health Sci. Ctr.

Director of Minimally Invasive Urol. Oncology, UCHSC

Denver, Colorado, USA

E-mail:fernando.kim@dhha.org

\title{
Comparison of Laparoendoscopic Single-site Donor Nephrectomy and Conventional Laparoscopic Donor Nephrectomy: Donor and Recipient Outcomes
}

Afaneh C, Aull MJ, Gimenez E, Wang G, Charlton M, Leeser DB, Kapur S, Del Pizzo JJ

Department of Surgery, Division of Transplantation Surgery, New York Presbyterian Weill Cornell Medical College, New York, New York

Urology. 2011; 78: 1332-7

Objective: To present a comparison of perioperative donor outcomes and recipient graft function in a series of patients undergoing laparoendoscopic single-site donor nephrectomy (LESS-DN) versus conventional laparoscopic donor nephrectomy (LDN).

Methods: Data were collected for 50 consecutive LESS-DN patients and a matched cohort of 50 LDN patients. The donor outcomes analyzed included operative time, estimated blood loss, complications, visual analog pain scores, and recovery time. The recipient outcomes analyzed included serum creatinine at discharge and follow-up and the incidence of delayed graft function.

Results: The mean total operative time was shorter in the LDN group than in the LESS-DN group (P < 0.0001). Linear regression analysis of the LESS-DN operative times relative to case number showed a significant decrease in the operative time with increasing case number $(\mathrm{r}(2)=0.19, \mathrm{P}=0.002)$. No statistically significant differences were found in estimated blood loss, warm ischemia time, length of stay, or visual analog pain scores between the 2 groups. However, the surgical incision was significantly smaller in the LESS-DN group $(\mathrm{P}<0.0001)$. After discharge, the patient-reported time to complete recovery was faster in the LESS$\mathrm{DN}$ group $(\mathrm{P}=0.01)$. The incidence of complications was similar in both groups; however, major complications only occurred in the LDN group. No differences were found in the recipient serum creatinine values or the incidence of delayed graft function.

Conclusion: Our initial experience with LESS-DN is encouraging. This retrospective matched-pair comparison between LESS-DN and LDN suggests that the single-port approach might be associated with quicker convalescence. Longer operative times in the LESS-DN group could simply represent the learning curve of a novel procedure. 


\title{
Editorial Comment
}

Laparoscopic live donor nephrectomy (LDN) has become the standard of care at most major academic centers. The benefits of laparoscopic over open donor nephrectomy have extensively been demonstrated since the first report by Kavoussi et al. Recently, the LESS Urological procedures are gaining popularity.

The authors studied 50 consecutive LESS-DN patients versus a matched cohort of 50 LDN patients. They demonstrated that LESS-DN patients recovered faster and complications were comparable with equal graft function and warm ischemia time. This report is valuable since convalescence and recovery is pivotal variables that may influence the decision to become a kidney donor increasing the pool of donors for the current high demand. The Gelport was used in this study that may also facilitate the learning curve compared to other single ports.

Dr. Fernando J. Kim

Chief of Urology, Denver Health Med. Ctr. Associate Professor, Univ. Colorado Health Sci. Ctr. Director of Minimally Invasive Urol. Oncology, UCHSC

Denver, Colorado, USA

E-mail:fernando.kim@dhha.org

\section{IMAGING}

\author{
Characterization of adrenal masses with diffusion-weighted imaging \\ Sandrasegaran K, Patel AA, Ramaswamy R, Samuel VP, Northcutt BG, Frank MS, Francis IR \\ Department of Radiology, Indiana University School of Medicine, Indianapolis, 46202, USA \\ AJR Am J Roentgenol. 2011; 197: 132-8
}

Objective: The purpose of this article is to assess the role of diffusion-weighted MRI in characterizing adrenal masses.

Materials and Methods: A retrospective review of the MRI database from August 2007 to July 2009 was performed. The MRI examinations of 48 patients, with 49 lesions, were reviewed independently and blindly by two experienced abdominal radiologists who measured the signal intensities on in-phase and opposed-phase T1-weighted imaging and apparent diffusion coefficient (ADC). ADC measurements and quantitative parameters of chemical shift imaging (signal intensity index and adrenal-to-spleen ratio) were assessed separately and in combination. Lesions with indeterminate signal intensity index $(<16.5 \%)$ were considered benign if ADC was greater than or equal to $1.0 \times 10(-3) \mathrm{mm}(2) / \mathrm{s}$ and malignant if ADC was less than $1.0 \times 10(-3) \mathrm{mm}(2) / \mathrm{s}$. Stepwise logistic regression analysis and receiver operating characteristic curves analysis were performed. Results: There were 12 malignant and 37 benign lesions. On multivariate analysis, the only significant predictors of lesion status were signal intensity index from reviewer $2(p=0.05)$ and lesion size $(p=0.04)$; ADC values were not found to be useful. On receiver operating characteristic curve analysis, there was no significant difference in area under the curve for ADC, signal intensity index, adrenal-to-spleen ratio, or the combined signal intensity index and ADC assessment. For lesions that were indeterminate according to signal intensity index, ADC values greater than $1.50 \times 10(-3) \mathrm{mm}(2) / \mathrm{s}$ were found only in benign lesions, and nine of 11 lesions with ADC less than $1.0 \times 10(-3) \mathrm{mm}(2) / \mathrm{s}$ were malignant. 